\title{
In this issue
}

Chen Chen, Xun Hou and Jinhai Si

Design of an integrated optics for transglutaminase conformational change

https://doi.org/10.1515/ntrev-2018-0022

Nanotechnol Rev 2018; 7(4): 283-290
Regular article: A novel integrated optics is performed in monitoring $\mathrm{TTG}$ and $\mathrm{Ca}^{2+}$ interaction and characterizing tTG conformation.

Keywords: dual polarizations; finite-element method; integrated optics; protein conformational change.

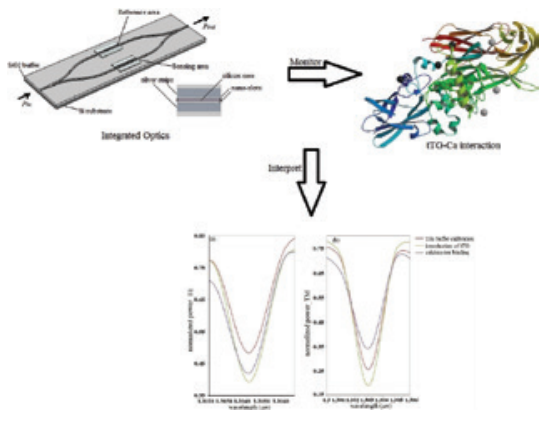

Zepeng Jiao, Bin Zhang, Chunya Li, Weicong Kuang, Jingxian Zhang, Yongqiang Xiong, Shaozao Tan, Xiang Cai and Langhuan Huang

Carboxymethyl cellulose-grafted graphene oxide for efficient antitumor drug delivery

https://doi.org/10.1515/ntrev-2018-0029 Nanotechnol Rev 2018; 7(4): 291-301
Regular article: Carboxymethyl cellulose-grafted graphene oxide (CMC-GO) was synthesized for loading anticancer drug methotrexate (MTX). The as-prepared drug delivery system MTX/CMC-GO demonstrated low toxicity and superior antitumor activity.

Keywords: cancer therapy;
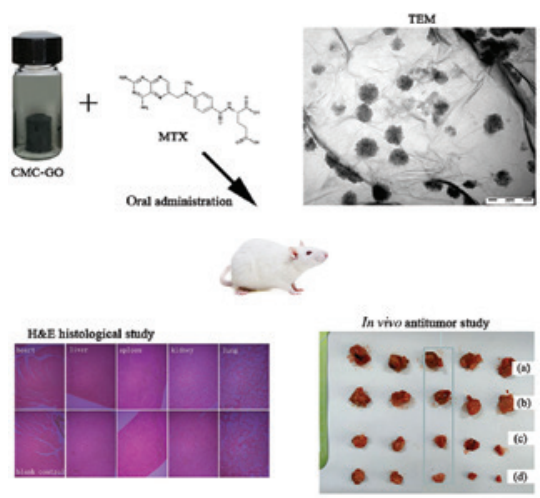
carboxymethyl cellulose; colorectal liver metastasis; drug delivery; graphene oxide.
Mahendra Rai, Avinash P. Ingle, Raksha Pandit, Priti Paralikar, Sudhir Shende, Indarchand Gupta, Jayanta K. Biswas and Silvio Silvério da Silva Copper and copper nanoparticles: role in management of insect-pests and pathogenic microbes

https://doi.org/10.1515/ntrev-2018-0031 Nanotechnol Rev 2018; 7(4): 303-315
Review: Copper is one of the important micronutrients required for plant growth. Moreover, the nano form of copper, i.e. copper nanoparticles (CuNPs) significantly promotes the plant growth as their small size facilitates easy uptake, penetration, and translocation in the plant cell. In addition, CuNPs also help in the management of insect-pests and microbes.

Keywords: agriculture; copper nanoparticles; plant nutrition; plant protection; toxicity.

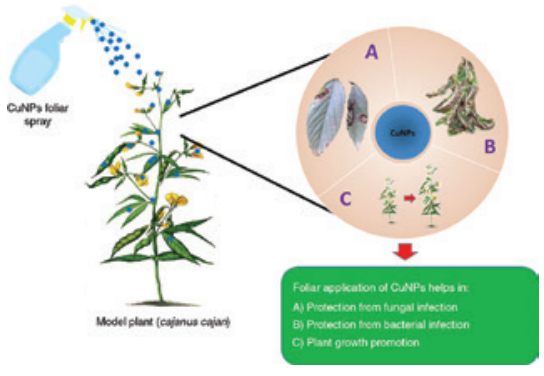


Gayathri Kandasamy, Vadim Annenkov and Uma Maheswari Krishnan

Nanoimmunotherapy - cloaked defenders to breach the cancer fortress

https://doi.org/10.1515/ntrev-2018-0013

Nanotechnol Rev 2018; 7(4): 317-340

Review: Graphical representation of the multimodal functional nanomaterials in combination with immunotherapy gives better efficacy in the treatment of cancer by triggering the

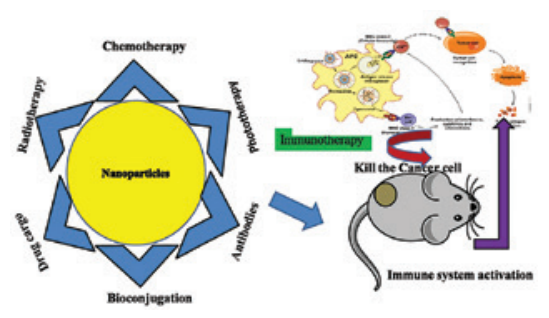
invoked immune response.

Keywords: cancer;

immunotherapy; nanoparticles.

Zhang Zhe and An Yuxiu

Nanotechnology for oil and gas industry an overview of recent progress

https://doi.org/10.1515/ntrev-2018-0061

Nanotechnol Rev 2018; 7(4): 341-353
Review: Nanotechnology has a huge potential to bring about revolutionary changes to many aspects of the energy industry. Nanomaterials acquire unique mechanical, chemical, and magnetic properties; therefore, they show superior performance over conventional micro and macro materials in a range of oil and gas field applications. Here, recent developments on the research of nanotechnology in drilling, completion, reservoir protection, enhance-oil-recovery (EOR), sensing and imaging techniques, stimulation techniques in oil and gas migration and accumulation has been summarized. Our aim was to provide a comprehensive scientific progress of nanotechnology in the oil and gas research area, identifying the existing barriers and challenges, and evaluating the technical and economic prospects in this field.

Keywords: drilling; EOR; nanotechnology. 